\title{
Colonic mucus: secretion and turnover in relation to dietary fibre intake
}

\author{
Iain A. Brownlee ${ }^{*}$, Mike E. Havler ${ }^{2}$, Peter W. Dettmar ${ }^{2}$, Adrian Allen ${ }^{1}$ and Jeffrey P. Pearson ${ }^{1}$ \\ ${ }^{I}$ Department of Physiological Sciences, Medical School, University of Newcastle upon Tyne, \\ Newcastle upon Tyne NE2 4HH, UK \\ ${ }^{2}$ Reckitt Benckiser Healthcare (UK) Ltd, Dansom Lane, Kingston upon Hull HU8 7DS, UK
}

\begin{abstract}
The colon is subjected to a myriad of potentially damaging agents that may reside within the lumen for $1-2 \mathrm{~d}$. Its first line of defence against these agents is the protective mucus bilayer that lines the entire colonic mucosa. This bilayer acts as a physical barrier to mucosal aggressors and also reduces shear stress to the mucosa. These actions are dependent on the unstimulated ('resting') colonic mucus thickness, and also on the rate that this layer can be replenished. The colonic mucus layer is altered in a number of colonic diseases that have been linked to a deficiency of fibre in the diet. The action of fibre intake on colonic mucus thickness and secretion is unknown. Using an in vivo rat model it has been demonstrated that: (1) fibre deficiency leads to a decreased protective potential of the mucus layer (e.g. the mean resting mucus thickness of the fibre-deficient group $(429 \mu \mathrm{m})$ was significantly lower than its respective control $(579 \mu \mathrm{m}$; $P<0.001)$, as was its total mucus secretion over $6 \mathrm{~h}(270 \mu \mathrm{m} v .541 \mu \mathrm{m} ; P<0.01)$; (2) specific fibre types in the diet alter the secretion dynamics of colonic mucus (e.g. a cellulose-based diet reduces total mucus secretion over $6 \mathrm{~h}$ compared with its control $(175 \mu \mathrm{m} v .463 \mu \mathrm{m})$. Analysis of the diets suggested a necessity for both soluble and insoluble fibre types in the diet to increase mucosal protection.
\end{abstract}

\section{Dietary fibre: Colonic mucus: Mucosal protection}

Dietary fibre intake has been linked to colonic disease for approximately 50 years (Burkitt, 1952). The mechanism of this association is not well understood. A wealth of evidence exists for the effects of dietary fibres on decreasing toxicity of colonic lumen contents in a variety of ways (Harris et al. 1998; Karakaya \& Kavas, 1999; Blackwood et al. 2000), as well as their beneficial interactions with lower bowel bacteria (Bengmark, 2000; Roberfroid, 2001).

\section{The colonic mucus barrier}

The mucosa expends large amounts of energy to produce a protective covering of mucus (synthesis of each mucin molecule requires $>43000$ ATP molecules). Recent in vivo studies have shown the mucus layer to be a bilayered system that protects the mucosa through two mechanisms (Strugala et al. 1999). The outer layer, which can be removed by suction has been termed the non-adherent mucus layer (Brownlee et al. 2002). This layer is believed to be vital in reducing shear stress to the mucosa (Allen et al. 1988). The constant removal of this weak gel layer in vivo may also act to trap mucosal aggressors (e.g. bacteria, endogenous proteases, secondary bile acids) and return them to the lumen. The underlying adherent layer (which cannot be removed by suction) acts as a sizeexclusion barrier to damaging luminal agents. This barrier must still allow the colonic uptake of water, salt and shortchain fatty acids (the absorptive functions of the colon), while at the same time preventing access of larger molecules to the mucosa. This system is especially important in stopping digestion of the colonic wall by both host and bacterial enzymes. In effect, this layer can be seen as a selectively-permeable partition between the mucosa and the luminal contents.

Primary to mucus layer function is the unstimulated thickness ('resting' mucus thickness) and the rate at which the mucus layer may be replenished after removal. These values may also be interpreted as indices of mucosal health and function. 


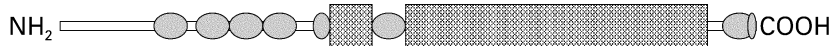

Fig. 1. MUC2 monomeric glycoprotein structure. ( domain; ( O-glycosylation regions. (Adapted from Allen et al. 1998; Desseyn et al. 2000; Dekker et al. 2002.)

\section{Mucus structure}

The functional component of mucus gels is mucin, a glycoprotein that makes up approximately $40 \mathrm{mg} / \mathrm{g}$ mucus wet weight (Allen et al. 1988). Four of the five known secretory mucins are localised to chromosomal location $11 \mathrm{p} \cdot 155$ (Desseyn et al. 2000; Dekker et al. 2002), including MUC2, which is the major mucin found in the colon. Secretory mucins share a number of similar structures that are directly related to their function (see Fig. 1).

The most obvious feature of mucins is the presence of a length of repeating protein sequence rich in threonine and/or serine and proline, referred to as the variable number tandem repeat (Allen et al. 1998). This repeat sequence is the major site for o-glycosylation on mucin molecules. In the human colon carbohydrate chains are of five to twelve sugars in length, and contain a relatively high ratio of negative sugars, thus endowing colonic mucus with an overall negative charge.

Key to the gel-forming properties of mucin molecules is their ability to polymerise. This process is mediated by a number of cysteine-rich areas at the $\mathrm{C}$ - and $\mathrm{N}$-termini of the protein core, allowing disulfide bridge formation.

The colonic mucus layer has been measured in vivo in rats and is approximately $500 \mu \mathrm{m}$ thick (Atuma et al. 2001), approximately $400 \mu \mathrm{m}$ of which is non-adherent mucus. Due to experimental limitations, similar measurements in human subjects are not possible. Mucus thickness measurements taken by conventional histology largely underestimate this thickness (for further details, see Strugala et al. 2003), and do not allow dynamic mucus secretion measurements to be made.

The aims of this ongoing study are: (a) to test whether inclusion of fibre in the diet has an effect on the mucus layer's protective potential in vivo; (b) to characterise the properties of dietary fibre that may be important in these effects.

\section{Methods}

Groups of ten male Wistar rats were each fed different fibrecontaining diets (approximately $140 \mathrm{~g}$ fibre $/ \mathrm{kg}$ diet) over 8 weeks. This study was done in two stages, each stage containing a control group (control 1 and 2 respectively) and two test diets. Table 1 shows the fibre content of each diet, as estimated by the Englyst procedure (Englyst \& Cummings, 1984).

During the feeding period body weight, faecal output and food and water intake were measured. Both food and water were available ad libitum. At the end of the feeding period the rats were starved overnight (16-17h). Colon mucus thickness measurements were subsequently made on terminally-anaesthetised rats using a previously described intravital microscopy technique (Atuma et al. 2001) over a
Table 1. Total fibre content $(\mathrm{g} / \mathrm{kg})$ of diets used in feeding trials*

\begin{tabular}{lccc}
\hline Diet & Soluble & Insoluble & Total \\
\hline Control 1 & $33 \cdot 8$ & $155 \cdot 2$ & 188.9 \\
Control 2 & $31 \cdot 7$ & 125 & $156 \cdot 7$ \\
Ispaghula & $130 \cdot 5$ & $21 \cdot 2$ & $151 \cdot 7$ \\
Pectin & $123 \cdot 2$ & $5 \cdot 9$ & 129 \\
Cellulose & $6 \cdot 1$ & $152 \cdot 8$ & 158.9 \\
Fibre-deficient & $2 \cdot 1$ & $2 \cdot 7$ & 4.8
\end{tabular}

* Total fibre content is given by summing the fibre content of the fractions that are not soluble in boiling phosphate buffer (insoluble), and those that are (soluble), using the methodology of Englyst \& Cummings (1984).

$6 \mathrm{~h}$ time course. During the first hour of measurement a plateau level of mucus thickness, termed the 'resting' mucus thickness, was observed. After $1 \mathrm{~h}$, the non-adherent layer was removed by suction, and further measurements taken. If the mucus layer returned to its 'resting' thickness over three consecutive readings (i.e. for $20 \mathrm{~min}$ ), then the suction was repeated, and measurements continued.

The adherent mucus thickness was taken as that which was left immediately after suction. By subtracting this value from the 'resting' thickness, the non-adherent thickness was calculated. Total mucus secretion was measured as mucus thickness increase per unit time. Statistical differences between the respective control groups and their test diets, as well as differences between test groups, were tested using one-way ANOVA and a Tukey post-test if significant differences $(P<0 \cdot 05)$ were present.

\section{Results \\ Resting thickness}

The colons of rats fed on cellulose and fibre-deficient diets had significantly lower resting mucus thicknesses than those for all other test diets $(P<0.01)$ and their respective controls $(P<0.001$; Fig. 2). The resting colon mucus thickness for the pectin diet was lower than that for the ispaghula diet $(P<0 \cdot 05)$, and for both diets they were lower than those for their respective control groups $(P<0 \cdot 05)$. Controls 1 and 2 were not significantly different (Fig. 2).

\section{Adherent thickness}

The rats fed on an ispaghula diet had a significantly thicker adherent layer than those fed on the fibre-deficient diet (205 $\mu \mathrm{m} v .154 \mu \mathrm{m} ; P<0 \cdot 05$; Fig. 3 ).

\section{Non-adherent thickness}

The fibre-deficient group had a significantly thinner nonadherent layer than control group $1(247 \mu \mathrm{m} v$. $375 \mu \mathrm{m}$; $P<0.05$; Fig. 4).

\section{Total mucus secretion}

On initial analysis the data could be divided into two groups, with pectin, cellulose and fibre-deficient diets all secreting relatively low levels of colonic mucus over time. The two controls and the ispaghula diet formed another group with a 

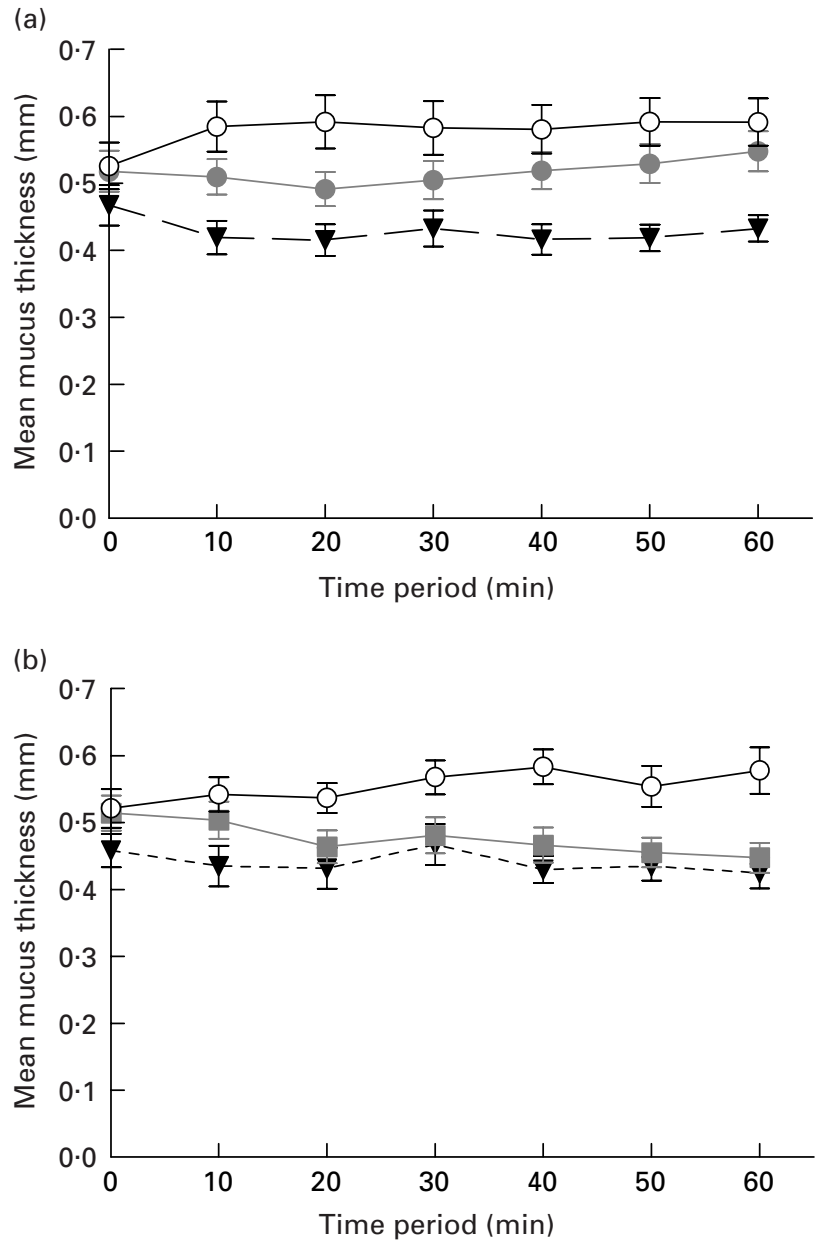

Fig. 2. Effect of dietary fibre type on mean resting colonic music thickness. (a) Effect of a fibre-deficient diet $(\boldsymbol{\nabla})$ and an ispaghulaenriched diet $(\bullet)$ on resting mucus thickness compared with a standard rodent diet (control 1; O). (b) Effect of pectin (w) and cellulose $(\boldsymbol{\nabla})$ compared with a standard rodent diet (control 2; $\bigcirc$ ). Values are means with their standard errors represented by vertical bars. For details of diets and procedures, see Table 1 and p. 246.

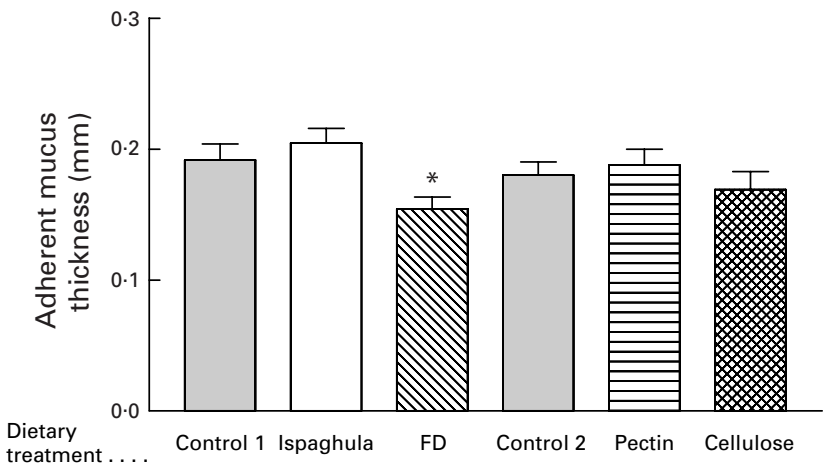

Fig. 3. Effect of dietary fibre type on mean adherent colonic mucus thickness. ( deficient (FD) diet; (E), pectin diet; ( $)$, cellulose diet. Values are means with their standard errors represented by vertical bars. For details of diets and procedures, see Table 1 and p. 246. The adherent colonic mucus thickness for the FD diet was significantly lower than that for the ispaghula diet: * $P<0.05$.

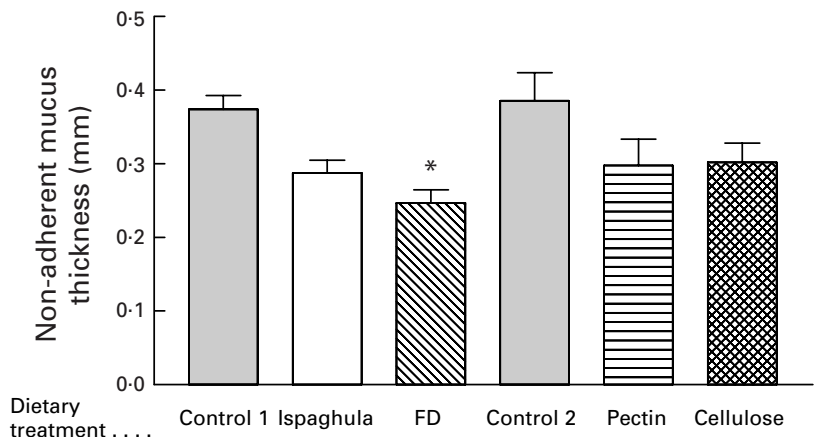

Fig. 4. Effect of dietary fibre type on mean non-adherent colonic mucus thickness. ( $)$, Control diets 1 and $2 ;(\square)$, ispaghula diet; (बw), fibre-deficient (FD) diet; (三), pectin diet; ( $)$, cellulose diet. Values are means with their standard errors represented by vertical bars. For details of diets and procedures, see Table 1 and p. 246. The nonadherent colonic mucus thickness for FD diet was significantly lower than that for its control (control 1 ): ${ }^{*} P<0.05$.

higher total mucus secretion. The animals fed on fibredeficient, cellulose- or pectin-enriched diets had significantly less $(P<0.01)$ total mucus secretion than their respective control group (control 1 for fibre-deficient, control 2 for cellulose and pectin) and the ispaghula-fed group (Fig. 5).

\section{Discussion}

The fibre-deficient, pectin and cellulose groups all displayed a decreased protective potential in their colonic mucus layer. This decreased potential was demonstrated by a thinner resting mucus thickness and a decreased secretory response. The two diets with relatively high levels of both soluble and insoluble fibres (i.e. the controls ( 1 and 2 ) and ispaghula; see Table 1) demonstrated a thicker resting mucus layer and a colonic mucosa with an enhanced ability to replace mucus. The modulation of colonic barrier function by dietary fibre may be the key to its role in decreasing colonic mucosal disease, although the mechanism is unclear. Previous evidence has suggested there could be a direct intermolecular interaction between certain dietary fibre types and colonic mucin in vitro (Brownlee et al. 2001), and this interaction could lead to a thickening of the mucus barrier in vivo.

In the colon there are a number of other areas where fibre in the diet could have an effect on the colonic mucus layer. Complex carbohydrates from dietary fibre could be absorbed via colonic antigen-presenting cells, and result in mucus production stimulation.

An increased distension of the colonic wall is known to bring about increased smooth muscle activity (Shafik et al. 2002). Mucus production could also be triggered and sustained by the same mechanism, in order to ease the passage of faeces along the colon and decrease the risk of mucosal damage.

Another key factor in colonic health is the microflora that reside in the colon. These micro-organisms metabolise undigested products (i.e. dietary fibre) in the colon. If there is an absence of fibre in the diet, or if the bacteria cannot metabolise the fibre in the diet (e.g. cellulose), then it is 


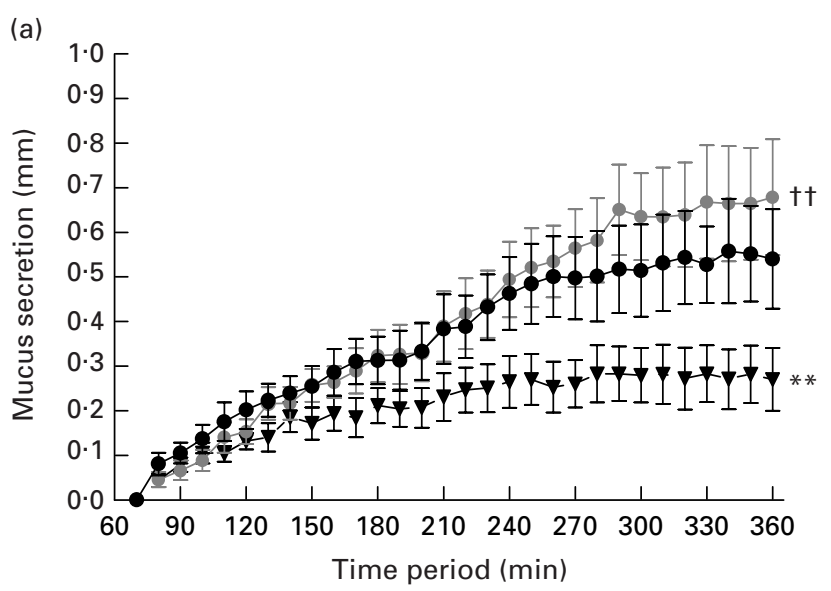

(b)

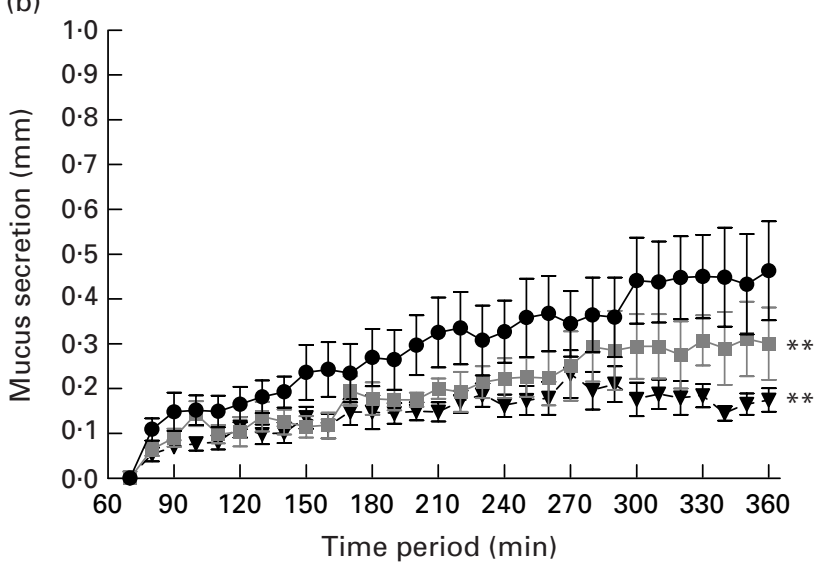

Fig. 5. Effect of dietary fibre type on mean colonic mucus replenishment rates. Values are means with their standard errors represented by vertical bars. For details of diets and procedures, see Table 1 and p. 246. (a) Effect of a fibre-deficient (FD) diet ( $\boldsymbol{\nabla}$ ) and an ispaghulaenriched diet $(\bullet)$ on mucus secretion compared with a standard rodent diet (control $1 ; \bullet$ ). The replenishment rate for the FD diet was significantly lower than that for its control (control 1): ${ }^{* *} P<0 \cdot 01$. (b) Effect of pectin ( and cellulose $(\boldsymbol{\nabla})$ compared with a standard rodent diet (control 2; $\boldsymbol{\bullet}$ ). The replenishment rates for the cellulose and pectin diets were significantly lower than that for their control (control 2): ${ }^{* *} P<0.01$. The replenishment rates for all other test (i.e. not control) diets were significantly lower than that for ispaghula: †† $P<0.01$.

possible that bacteria that live on the other major energy sources in the colon (e.g. the mucus layer) will begin to thrive. This situation could lead to increased rates of mucus degradation, linked to a less-well-protected mucosa, resulting in bacterial access to the mucosa.

Some products of bacterial metabolism have been shown to be beneficial to the colon in a number of ways (McIntyre et al. 1993; Ichikawa \& Sakata, 1997). The absence of a fermentable substrate would lead to decreased levels of these compounds, potentially leading to a lumen environment that is more aggressive to the mucosa.

Dietary fibre type has also been linked to increased binding of potentially-damaging agents in the colonic lumen (Owen, 1997; Harris et al. 1998; Jenkins et al. 2001).
Potentially, an increase in the number, or a change in types, of dietary fibres might lead to binding of a wider range of lumen mucosal aggressors, thereby decreasing the exposure time of the colonic mucosa to these agents.

Overall, our results suggest that inclusion of substantial amounts of both soluble and insoluble fibre types in the diet benefits colonic health by increasing the protective properties of the colonic mucus layer.

\section{Future work}

Using the same model described in the present paper, the effects of a number of different dietary fibres on the colonic mucus layer as a means of measuring colonic health can be tested. This investigation, together with a study of the biochemical and biophysical properties of these complex carbohydrates, will make it possible to define the properties of dietary fibre that are most important to colonic well-being.

It is essential to understand the interactions between all the elements involved in colonic health and disease. To achieve this objective is not a minor undertaking, and requires the expertise in a number of fields that can only be achieved through a multidisciplinary approach at a number of research centres. Due to our links with the Reckitt Benckiser Multicentre Lower Bowel Research network, we can currently carry out such a research programme.

\section{References}

Allen A, Hutton DA \& Pearson JP (1998) The MUC2 gene product: a human intestinal mucin. International Journal of Biochemistry and Cell Biology 30, 797-801.

Allen A, Leonard AJ \& Sellars LA (1988) The mucus barrier - its role in gastroduodenal mucosal protection. Journal of Clinical Gastroenterology 10, S93-S98.

Atuma C, Strugala V, Allen A \& Holm L (2001) The adherent gastrointestinal mucus gel layer: thickness and physical state in vivo. American Journal of Physiology 280, G922-G929.

Bengmark S (2000) Colonic food: Pre- and probiotics. American Journal of Gastroenterology 95, S5-S7.

Blackwood AD, Salter J, Dettmar PW \& Chaplin MF (2000) Dietary fibre, physicochemical properties and their relationship to health. Journal of the Royal Society for the Promotion of Health 120, 242-247.

Brownlee I, Havler ME, Allen A, Dettmar PW \& Pearson JP (2002) A differential effect of dietary fibre on protection of the colonic mucosa. Journal of Gastroenterology and Hepatology 17, Suppl., A576.

Brownlee IA, Allen A, Dettmar PW \& Pearson JP (2001) Mucosal protective properties of dietary fibre may be mediated through an interaction with mucus. Gut 49, Suppl. III, 2440.

Burkitt DP (1952) Acute abdomens: British and Baganda compared. East African Medical Journal 29l, 189-194.

Dekker J, Rossen JWA, Buller HA \& Einerhand AWC (2002) The MUC family: an obituary. Trends in Biochemical Sciences 27, 126-131.

Desseyn J-L, Aubert J-P, Porchet N \& Laine A (2000) Evolution of the large secreted gel-forming mucins. Molecular Biology and Evolution 17, 1175-1184.

Englyst HN \& Cummings JH (1984) Simplified method for the measurement of total non-starch polysaccharides by gas-liquid chromatography of constituent sugars as alditol acids. Analyst 109, 937-943. 
Harris PJ, Vallappilakkandy KS, Roberton AM, Triggs CM, Blakeney AB \& Ferguson LR (1998) Adsorption of a hydrophobic mutagen to cereal brans and cereal bran dietary fibres. Mutation Research 412, 323-331.

Ichikawa H \& Sakata T (1997) Effect of L-lactic acid, short-chain fatty acids, and $\mathrm{pH}$ in cecal infusate on morphometric and cell kinetic parameters of rat cecum. Digestive Diseases and Sciences 42, 1598-1610.

Jenkins DJA, Kendall CWC, Popovich EV, Mehling CC, Vuksan V, Ransom TPP, Rao AV, Rosenberg-Zand R, Tariq N, Corey P, Jones PJH, Raeini M, Story JA, Furumoto EJ, Illingworth DR, Pappu AS \& Connelly PW (2001) Effect of a very-high fiber vegetable, fruit and nut diet on serum lipids and colonic function. Metabolism 50, 494-503.

Karakaya S \& Kavas A (1999) Adsorption of direct-acting and indirect-acting mutagens by various dietary fibers. International Journal of Food Sciences and Nutrition 50, 319-323.
McIntyre A, Gibson PR \& Young GP (1993) Butyrate production from dietary fibre and protection against large bowel cancer in a rat model. Gut 34, 386-391.

Owen RW (1997) Faecal steroids and colorectal carcinogenesis. Scandinavian Journal of Gastroenterology 32, Suppl. 222, 7682.

Roberfroid MB (2001) Prebiotics: preferential substrates for specific germs? American Journal of Clinical Nutrition 73, 406S-409S.

Shafik A, El-Sibai O \& Ahmed I (2002) On the mechanism of colonic motility: The electric activation theory of colonic contraction. Journal of Surgical Research 103, 8-12.

Strugala V, Allen A, Dettmar PW \& Pearson JP (2003) Colonic mucin: methods of measuring mucus thickness. Proceedings of the Nutrition Society 62, 237-243.

Strugala V, Dettmar PW, Pearson JP \& Allen A (1999) Is the colonic barrier multilayered? Gastroenterology 116, G1395. 